\title{
Proteomics provides insights into biological pathways altered by plant growth promoting bacteria and arbuscular mycorrhiza in sorghum grown in marginal soil
}

Faten Dhawi ${ }^{\mathrm{a}, \mathrm{b}}$, Rupali Datta ${ }^{\mathrm{a}}$, Wusirika Ramakrishna ${ }^{a, \mathrm{c}}$

Running title: Sorghum proteins modulated by microbes 


\section{ABSTRACT}

Sorghum is an economically important crop, a model system for gene discovery and a biofuel source. Sorghum seedlings were subjected to three microbial treatments, plant growth promoting bacteria (B), arbuscular mycorrhizal (AM) fungi mix with two Glomus species (G. aggregatum and G. etunicatum), Funelliformis mosseae and Rhizophagus irregularis $(\mathrm{My})$, and $\mathrm{B}$ and $\mathrm{My}$ combined $(\mathrm{My}+\mathrm{B})$. Proteomic analysis was conducted followed by integration with metabolite, plant biomass and nutrient data. Out of 366 differentially expressed proteins in sorghum roots, 44 upregulated proteins overlapping among three treatment groups showed positive correlation with sorghum biomass or element uptake or both. Proteins upregulated only in B group include asparagine synthetase which showed negative correlation with biomass and uptake of elements. Phosphoribosyl amino imidazole succinocarboxamide protein with more than 50-fold change in $\mathrm{My}$ and $\mathrm{My}+\mathrm{B}$ groups correlated positively with $\mathrm{Ca}, \mathrm{Cu}, \mathrm{S}$ and sucrose levels in roots. The B group showed the highest number of upregulated proteins among the three groups with negative correlation with sorghum biomass and element uptake. KEGG pathway analysis identified carbon fixation as the unique pathway associated with common upregulated proteins while biosynthesis of amino acids and fatty acid degradation were associated with common downregulated proteins. Protein-protein interaction analysis using STRING identified a major network with thirteen downregulated proteins. These findings suggest that plant-growth-promoting-bacteria alone or in combination with mycorrhiza enhanced radical scavenging system and increased levels of specific proteins thereby shifting the metabolism towards synthesis of carbohydrates resulting in sorghum biomass increase and uptake of nutrients.

Keywords: Proteomics, Metabolites, Plant Growth Promoting Bacteria, Mycorrhiza, Pathways 


\section{Introduction}

One of the major challenges in biology is to understand how plant cells integrate external and internal signals during stress. Plants exposed to various types of abiotic stress such as drought, extreme temperatures, and nutrient deficient soil etc. are known to suppress their natural growth. Abiotic or biotic stress factors are known induce reactive oxygen species (ROS). Although ROS has been shown to perform diverse functions as signaling molecules, regulate cellular processes (Apel and Hirt, 2004) and contribute to intercellular communication (Jaillais and Chory, 2010), high levels of ROS can cause cellular imbalance. Excessive ROS interfered with lipid peroxidation (Shah et al. 2001), DNA synthesis (Brawn and Fridovich, 1981) and enzymatic activities (Fucciet et al. 1983). When ROS reached high levels, cellular scavenging system was activated via either ROS scavenging enzymes such as superoxide dismutase (SOD) (Alscher et al. 2002; Apel and Hirt, 2004), or non-enzymatic processes.

Plants grown on soil deficient in essential nutrients suffer from physiological disorders with negative effect on growth and development. The nutrient imbalance affected synthesis of essential molecules leading to disturbed biological processes (da Silva et al. 2010). The imbalanced biological system puts plants under stress causing photosynthetic impairment and increase in radical oxygen species (Sharma et al. 2012).

Plants under stress save energy by producing sugars and downregulating protein synthesis (Srivastava et al. 2013). Nutrient deficiency can be overcome by adding microbes such as plant growth promoting bacteria PGPB (Glick, 2012) or mycorrhizal fungi (Khan, 2005; Guo et al. 2015). The Pseudomonas sp. TLC 6-6.5-4 isolated from Torch Lake MI, enhanced availability of nutrients such as phosphorous and produced indole-3-acetic acid (IAA), a phytohormone, leading to higher maize biomass grown in nutrient-deficient soil (Li and Ramakrishna, 2011). The increase of plant plasticity by changing the soil rhizosphere with microbial interaction was reported in several studies (Duponnois et al. 2006; Gohre and Paszkowski, 2006). Field studies conducted with maize inoculated with AM fungi and PGPB not only increased plant biomass but also showed significant increase in grain weight, iron and zinc content and number of grains 
per spike (Berta et al. 2014). However, AM fungi and PGPB showed variable effects on the quality and quantity of grain starch and proteins.

The aim of the current study was to evaluate the effect of different microbial inoculations on sorghum root protein expression and their association with other physiological responses such as biomass and element uptake in nutrient-poor soil. Sorghum seedlings were grown in nutrient-deficient, sandy soil collected from Torch Lake, MI in the greenhouse in the presence of plant growth promoting bacteria (PGPB) alone, arbuscular mycorrhiza alone, PGPB with arbuscular mycorrhiza combined, and dead inoculum (control group). Analysis of sorghum root proteins showed that all of the three microbial treatments caused upregulation of proteins such as superoxide dismutase (SOD) involved in scavenging of reactive oxygen species (ROS). The PGPB group showed the highest number of upregulated proteins followed by $\mathrm{My}+\mathrm{B}$ and $\mathrm{My}$ groups. Several upregulated proteins associated with higher levels of specific metabolites were related to carbohydrate biosynthesis.

\section{Materials and Methods}

\subsection{Plant material and analysis}

Plant material and analyses were carried out as described in Dhawi et al. 2015. Three regimes were applied to sorghum BTx623 seedlings grown in stamp sand soil collected from Michigan Upper Peninsula (47.187518 latitude, 88.403788 longitude). The 48 hours old seedlings sown in stamp sand were subjected to three treatments: mycorrhiza mix alone (My), mycorrhiza mix + plant growth promoting bacteria Pseudomonas sp. TLC 6-6.5-4 (My+B) and Pseudomonas sp. TLC 6-6.5-4 alone (B). The PGPB strain was isolated from Torch Lake and used in a previous study with maize and sorghum ( $\mathrm{Li}$ and Ramakrishna, 2011; Dhawi et al. 2015; Dhawi et al. 2016). Each of My or My+B group contained $9 \mathrm{~g}$ of inoculum ( 1320 propagules) of mycorrhiza

suspended in $0.85 \%$ sodium chloride. The mycorrhiza mix had two Glomus species (aggregatum and etunicatum), Funelliformis mosseae and Rhizophagus irregularis (Valentine Country Inc., ND, USA). Each of B or $\mathrm{My}+\mathrm{B}$ group contained $10^{-8}$ cfu of Pseudomonas sp. TLC 6-6.5-4. The control group C was 
subjected to the same amount of dead inoculum as $\mathrm{My}+\mathrm{B}$ group. All the plants were grown in the green house with controlled temperature $\left(28^{0} \mathrm{C}\right)$ and humiditiy 65\%). Three months' later, sorghum roots were harvested for protein analysis. Biomass and elements analysis data was used from the previous study (Dhawi et al. 2016) to perform correlation analysis with protein expression data.

\subsection{Protein profiling}

Frozen roots, three samples for each group $\left(-80^{\circ} \mathrm{C}\right)$ were manually homogenized with liquid nitrogen and stored at $-80^{\circ} \mathrm{C}$. Protein extraction was modified from Fukao et al. (2011) method. Four hundred milligrams of each sample was used with $700 \mu$ of protein extraction buffer (50mM HEPES-KOH, pH 7.5, 5mM EDTA, $400 \mathrm{mM}$ sucrose, $0.1 \%$ Triton X-100 and protease inhibitor cocktail). Samples were sonicated in a cold bath for $45 \mathrm{~min}$ with vortexing every $10 \mathrm{~min}$, followed by centrifugation at $4{ }^{\circ} \mathrm{C}$ for $30 \mathrm{~min}$ at $13,000 \mathrm{~g}$. To the supernatant, $500 \mu \mathrm{l}$ of ice cold TCA/acetone (acetone 10\%) was added, followed by incubation for $45 \mathrm{~min}$ at $-20^{\circ} \mathrm{C}$ and centrifugation. The pellet was dissolved in $50 \mu \mathrm{l}$ of $0.5 \mathrm{M}$ triethylammonium bicarbonate (TEAB) with $0.2 \%$ SDS and stored at $80^{\circ} \mathrm{C}$. The extracted protein samples were sent to Keck Foundation Biotechnology Resource Laboratory at Yale University, for LC-MS/MS analysis. Protein samples (three samples for each group) were digested with trypsin and then analyzed using LC-MS/MS on Waters/Micromass AB QSTAR Elite (Waters nanoACQUITY ultra high pressure liquid chromatographs UPLC) for peptide separation. Detection and quantification of all peptide ions was followed by expression analysis and identification of proteins. Default normalization from Progenesis QI was utilized to obtain the quantitation value across the runs (http://www.nonlinear.com/progenesis/qi/v2.0/faq/how-normalisation-works.aspx). Protein abundances were calculated from these peptide abundances according to Progenesis QI (http://www.nonlinear.com/progenesis/qi-for-proteomics/v2.0/faq/howare-protein-abundances-calculated.aspx). The function of each protein was identified using UniProt (http://www.uniprot.org/). Analysis of proteins among groups was performed using a web based tool, InteractiVenn (Heberle et al. 2015). Protein levels of 1.2 fold in $\mathrm{B}, \mathrm{My}$, and $\mathrm{My}+\mathrm{B}$ groups in comparison to control were considered as significant. Positive and negative correlation was considered to be significant at $\geq 0.56$. 


\subsection{Analysis of biological pathways and protein-protein interactions}

Common upregulated and downregulated proteins were analyzed using STRING (Szklarczyk et al. 2015; strings-db.org/cgi/network.pl) to identify protein-protein interactions and proteins that are part of KEGG pathways. The initial cut-off false discovery rate used for identifying upregulated and downregulated proteins association with KEGG pathway was 0.05 . Only proteins that matched Sorghum bicolor proteins were identified and used for protein-protein interaction analysis. A false discovery rate cut-off of 0.01 was used in the final output analysis to report KEGG pathways associated with these proteins in Table 1. Each node in a network represents an identified protein with three-dimension structure and each network edge represents an interaction.

\section{Results}

\subsection{Different patterns shown by upregulated proteins in treatment groups}

Proteomic analysis identified 787 proteins in sorghum roots. Among these proteins, 421 were annotated as putative uncharacterized Sorghum bicolor proteins and 255 plantcharacterized proteins including 40 redundant plant-related proteins (Fig. 1). In addition, 57 fungus related proteins were identified with 12 of them annotated as putative uncharacterized (Fig. S1). The total number of bacterial related proteins were 54 (Fig. S2). Out of these, significant upregulation of 96 proteins in B group, 86 proteins in $\mathrm{My}+\mathrm{B}$ group and 72 proteins in My group in comparison with control group was observed. The upregulation of 44 proteins was common among the three groups with 14 and 15 proteins common between $\mathrm{B}$ and $\mathrm{My}+\mathrm{B}$, and between My and $\mathrm{My}+\mathrm{B}$ groups, respectively (Fig. S3).

Twenty nine proteins (13.5\% of total upregulated proteins) were upregulated exclusively in B, 13 (6\%) in My+B and (1.4\%) in My group. Functional classification showed that the highest number of proteins were related to oxidoreductase activity which reached 19\%, followed by amino acids biosynthesis (9.3\%) (Fig. 2). In comparison, My group had the same total number of proteins with $\mathrm{My}+\mathrm{B}$ group related to functions such 
as carbohydrate synthesis and a lower number of proteins involved in amino-acid biosynthesis. Some other proteins had minor or no differences among the three groups (B, My and $\mathrm{My}+\mathrm{B}$ ) such as proteins related to energy production, transferase, metabolism of nitrogen and cell cycle regulation.

Analysis of proteins upregulated in all three treatments identified 5.3, 4 and 2.4 fold average upregulation in $\mathrm{My}, \mathrm{My}+\mathrm{B}$ and B groups, respectively. Some proteins such as mitogen activated protein kinase (>11 fold), putative IQ calmodulin-binding domaincontaining family protein ( $>5$ fold) are upregulated in all treatment groups. The upregulated proteins showed similar tendency for the two groups, My and My+B. For instance, phosphoribosyl aminoimidazole-succinocarboxamide synthase (PAICS) showed highest upregulation ( $>50$ fold) in My and $\mathrm{My}+\mathrm{B}$ groups while it was upregulated only 2 fold in B group. Similarly, dihydrolipoyl dehydrogenase (>9 fold) and proliferating cell nuclear antigen (PCNA; >5 fold) were highly upregulated in My and My+B groups compared to B group where only 2 fold upregulation was observed. Cysteine synthase with 2, 5 and 7 fold levels in B, My+B and My groups, respectively, and nucleolar protein 58 (NP58) with 2, 3, and 6 fold in B, My+B and My groups, respectively, in comparison to control showed a similar trend.

\subsection{Proteins upregulated with microbial treatments correlated with biomass and element} uptake in sorghum root

All three treatments resulted in highly significant increase in shoot and root biomass (Dhawi et al. 2015). However, PGPB treated plants (B group) showed the best response with reference to increase in both shoot and root biomass. Most of the upregulated proteins common among the three groups $(\mathrm{My}, \mathrm{B}$ and $\mathrm{My}+\mathrm{B})$ were found to be correlated with biomass and or element uptake. Thirty-seven proteins showed positive correlation with biomass and or uptake of elements and six proteins showed negative correlation (Table S1). Correlation analysis identified four proteins, putative heat shock protein 2, superoxide dismutase, mitogen-activated protein kinase and fructokinase to be positively correlated with shoot and root biomass. In addition, three proteins, glyceraldehyde-3-phosphate dehydrogenase, 14-3-3-like protein (GF14-C) and triose

phosphate isomerase, correlated negatively with shoot and root biomass. Asparagine 
synthetase (glutamine-hydrolyzing), which was unique to B group showed a negative correlation with biomass increase.

All the ten elements (P, K, Ca, Mg, Mn, Zn, Fe, Al, Cu and $\mathrm{S}$ ) in shoot and root showed positive correlation with superoxide dismutase and negatively with two proteins, glyceraldehyde-3-phosphate dehydrogenase 1 and asparagine synthetase (glutaminehydrolyzing). The same ten elements in shoot ( $\mathrm{P}, \mathrm{K}, \mathrm{Ca}, \mathrm{Mg}, \mathrm{Mn}, \mathrm{Zn}, \mathrm{Fe}, \mathrm{Al}, \mathrm{Cu}$ and S) correlated positively with upregulation of superoxide dismutase (SOD) in all three groups. Some elements, $\mathrm{Ca}$ and root $\mathrm{P}$ and $\mathrm{K}$ correlated positively with upregulation of proliferating cell nuclear antigen (PCNA). Correlation analysis identified fructokinase to be correlated positively with primary nutrients, $\mathrm{P}$ and $\mathrm{K}$ in root and shoot and six proteins that are negatively correlated with four of them (fructokinase-2, 14-3-3-like protein [GF14-C], glyceraldehyde-3-phosphate dehydrogenase 1 and triosephosphate isomerase) common between groups and two of them, putative BRE1 E3 ubiquitin ligase and asparagine synthetase (glutamine-hydrolyzing) upregulated in B group.

Upregulated proteins that correlated positively with primary nutrients $(P, K)$ in root were 31. Among these proteins, 29 were common in three groups and monodehydroascorbate reductase (MDHAR) was found only in B and solanesyl diphosphate synthase 1 was found only in My group. The macro elements, $\mathrm{Ca}$ and $\mathrm{Mg}$ in shoot, and $\mathrm{Ca}$ and $\mathrm{S}$ in root correlated negatively with three proteins, fructokinase, putative ribosomal protein L3 and malic enzyme. Triosephosphate isomerase correlated negatively with sorghum biomass, macroelements $(\mathrm{Ca}, \mathrm{Mg}$ and $\mathrm{S})$, primary elements $(\mathrm{P}$ and $\mathrm{K})$, microelements ( $\mathrm{Mn}$ and $\mathrm{Fe}$ ), and heavy metals ( $\mathrm{Cu}$ and $\mathrm{Al})$ in shoot and root. Triosephosphate isomerase correlated negatively with two metabolites (mucic acid and ferulic acid) known to correlate positively with element uptake. $\mathrm{Ca}$ in shoot and root correlated positively with 31 proteins with one of them, MDHAR upregulated in B group only. The same element $(\mathrm{Ca})$ in shoot and root correlated negatively with 18 proteins with 6 of them upregulated in B group while three were upregulated in $M y+B$ group. Additionally, $\mathrm{P}$ and $\mathrm{K}$ in root correlated positively with 31 proteins with solanesyl diphosphate synthase 1 upregulated in My group only and MDHAR upregulated in B group only. Heavy metals ( $\mathrm{Zn}, \mathrm{Cu}$ and $\mathrm{Al}$ ) in shoots and roots correlated positively with two proteins, bifunctional methylthioribulose-1-phosphate dehydratase/enolase- 
phosphatase E1 and phosphoglycerate kinase and correlated negatively with three proteins, elongation factor $1 \alpha, \mathrm{V}$-type proton ATPase and cytochrome P450. Moreover, copper in shoot and root correlated positively with 33 proteins and negatively with 35 proteins (data not shown) whereas, aluminum in shoots and roots correlated positively with three proteins (bifunctional methylthioribulose-1-phosphate dehydratase/enolasephosphatase E1, 26S protease regulatory subunit 8 homolog $\mathrm{A}$ and phosphoglycerate kinase) and negatively with seven proteins (elongation factor $1 \alpha$, V-type proton ATPase, cytochrome P450, glutamate dehydrogenase, pyruvate dehydrogenase E1 component subunit $\alpha$, carboxypeptidase and glyceraldehyde-3-phosphate dehydrogenase). Nucleolar protein, NP58 correlated positively with uptake of microelements (Mn and Fe) and heavy metals $(\mathrm{Cu}$ and $\mathrm{Al})$ in shoot and root. MDHAR upregulated in B group only, correlated positively with shoot $\mathrm{Ca}$ and root $\mathrm{Ca}, \mathrm{P}$ and $\mathrm{K}$ and two proteins, glutamate dehydrogenase and asparagine synthetase correlated negatively with biomass and uptake of elements. In addition to the above proteins, ROS scavenging enzyme, SOD and a peroxidase are upregulated in all three treatments

\subsection{Upregulated proteins associated with metabolites, biomass and element uptake}

Only metabolites that showed a positive correlation with biomass and element uptake were used in correlation analysis with upregulated proteins among the three groups. Fructokinase correlated positively with metabolites, stearic acid, sorbitol and sebacic acid which in turn showed positive correlation with biomass and element uptake (Table S1). The same metabolites correlated negatively with glyceraldehyde-3-phosphate dehydrogenase. PAICS correlated positively with root $\mathrm{Ca}, \mathrm{S}, \mathrm{Cu}$ and sucrose, while negatively with proline, methionine and lysine in our study. Upregulation of putative ribosomal protein L3 (RPL3) and malic enzyme correlated positively with macroelements ( $\mathrm{Ca}, \mathrm{Mg}$ and $\mathrm{S}$ ) and $\mathrm{P}$ and $\mathrm{K}$ in root. On the other hand, two metabolites, mucic acid and ferulic acid which correlated positively with uptake of nine elements ( $\mathrm{P}, \mathrm{K}, \mathrm{Ca}, \mathrm{Mg}, \mathrm{Mn}$, $\mathrm{Zn}, \mathrm{Fe}, \mathrm{Al}, \mathrm{Cu}$ and $\mathrm{S}$ ) showed positive correlation with five proteins (histone $\mathrm{H} 2 \mathrm{~B}$, cysteine synthase, superoxide dismutase, aquaporin TIP2-1 and 26S protease regulatory subunit 8 homolog A). These two metabolites correlated negatively with seven proteins, glyceraldehyde-3-phosphate dehydrogenase 1, triosephosphate isomerase, fructokinase-2, 
14-3-3-like protein (GF14-C), glyceraldehyde-3, phosphate dehydrogenase 3, cytosolic with two of them, heat shock cognate $70 \mathrm{kDa}$ protein 3 and sensor protein LytS upregulated exclusively in My+B group.

\subsection{Downregulated proteins and their correlation with biomass and element uptake}

The total downregulated proteins were 226 in B group, 222 in My group and 206 in $\mathrm{My}+\mathrm{B}$ group. Among these proteins, 9 proteins overlapped only between $\mathrm{My}$ and $\mathrm{My}+\mathrm{B}$ groups, 28 proteins between B and $\mathrm{My}+\mathrm{B}$ groups and 35 proteins between My and B groups (Fig.S4). A total of 153 proteins common among the three groups (B, My and $\mathrm{My}+\mathrm{B})$ were downregulated. Average downregulation of proteins was highest in My group ( 4.5 fold) followed by $\mathrm{My}+\mathrm{B}$ (2.6 fold) and My (1.5 fold) groups. This pattern was similar to that observed for upregulated proteins.

Malate dehydrogenase 2 showed 30, 7 and 3 fold downregulation in $\mathrm{My}, \mathrm{My}+\mathrm{B}$ and B groups, respectively followed by aquaporin PIP (Plasma membrane intrinsic protein) 1-1 which was downregulated 27, 5, and 2 fold in $\mathrm{My}, \mathrm{My}+\mathrm{B}$ and B groups, respectively (Fig. S5). Aquaporin PIP1 downregulation showed negative correlation with biomass, uptake of elements and several metabolites (Table S1). Citrate synthase which was the most downregulated (4 fold) protein in B group was 24 and 4 fold downregulated in My and My+B groups, respectively, correlated negatively with root uptake for $\mathrm{P}, \mathrm{Ca}, \mathrm{S}$ and $\mathrm{Cu}$. In contrast, hexokinase-5 which showed 18, 4, and 3 fold downregulation in My, $\mathrm{My}+\mathrm{B}$ and $\mathrm{B}$ groups, respectively, correlated positively with the uptake of the same elements in sorghum root. Regulatory protein, RecX involved in recombination (36 fold), ATP-dependent Clp protease (12 fold) and Dof21, a transcription factor (8 fold) were highly downregulated in $\mathrm{My}+\mathrm{B}$ group. In contrast, the first two proteins were downregulated about 4 fold whereas Dof 21 showed 12 fold downregulation in My group and relatively less downregulation in B group.

Functional classification of proteins showed that most of the downregulated proteins were related to oxidoreductase activity (16\%), translation elongation (13\%) and transport activities (10\%) with a slight difference among the three groups (Fig. S6). In comparison, a higher percentage (19\%) of upregulated proteins were related to oxidoreductase activity. 


\subsection{Biological pathways and protein-protein interactions of common upregulated and}

downregulated proteins

The common upregulated proteins analyzed using STRING identified triose phosphate isomerase, malic enzyme, glucose-6-phosphate isomerase, dihydrolipoyl dehydrogenase and peroxidase) with significant involvement in five plant associated KEGG pathways (Table 1). Two of these proteins, triose phosphate isomerase and glucose-6-phosphate isomerase showed protein-protein interactions based on STRING analysis (Fig. S7). The analysis of downregulated proteins identified 12 proteins, namely, aconitate hydratase, acyl-coenzyme A oxidase, aldehyde dehydrogenase, $\beta$ hexosaminidase, chorismate synthase, citrate synthase, cytochrome P450, fructose bisphosphate aldolase, methylene tetrahydrofolate reductase, phenylalanine ammonia lyase, phosphoenolpyruvate carboxylase, and pyruvate kinase, which are involved in six plant associated KEGG pathways (Table 1). Six downregulated proteins, aconitate hydratase, citrate synthase, fructose bisphosphate aldolase, pyruvate kinase, phosphoenolpyruvate carboxylase, aldehyde dehydrogenase, associated with KEGG pathways showed protein-protein interactions which supports possible functional relationship among them (Fig. 4). Although four pathways, glycolysis/ gluconeogenesis, pyruvate metabolism, carbon metabolism and biosynthesis of secondary metabolites were associated with both up and downregulated proteins, the specific proteins which were up or downregulated were different. For instance, triose phosphate isomerase, dihydrolipoyl dehydrogenase, and glucose-6-phosphate isomerase were upregulated whereas pyruvate kinase, fructose bisphosphate aldolase, and aldehyde dehydrogenase were downregulated in glycolysis/ gluconeogenesis. The upregulated proteins showed unique association with carbon fixation in photosynthetic organisms whereas downregulated proteins showed unique association with fatty acid degradation and biosynthesis of amino acids. The common downregulated proteins associated with biological pathways showed a major network with thirteen proteins. Malate dehydrogenase 2 showed the highest number (8) of interacting proteins, namely, thioredoxin, glutathione peroxidase, aconitate hydratase, citrate synthase, glutamate dehydrogenase, fructose bisphosphate aldolase, pyruvate kinase, phosphoenolpyruvate carboxylase, ATP synthase subunit $\beta$, mitochondrial 
aldehyde dehydrogenase, putative alcohol dehydrogenase, and aldehyde dehydrogenase (Fig. 4). A second network with five interacting members belonged to those involved in protein synthesis. Overall, this analysis identified functional interactions which are biologically relevant among downregulated proteins.

\section{Discussion}

Growing plants in marginal soil increases the need for molecular adjustment to compensate for the lack of essential nutrients. In plants, reactive oxygen species (ROS) are necessary in low levels to provide a balanced cellular redox for growth regulation whereas high ROS levels cause cellular damage and death (Bailly et al. 2008). A number of proteins involved in reducing cellular ROS levels were upregulated in maize seeds inoculated with AM fungi under field conditions (Bona et al. 2016). SODs are metalcontaining enzymes with different types $(\mathrm{Fe}, \mathrm{Mn}$ and $\mathrm{Cu} / \mathrm{Zn}$ ) involved in first line defense against ROS under various abiotic stress conditions such as water and salt stress (Ramírez et al. 2012; Diaz-Vivancos et al. 2013). Under salt stress, transgenic plum plantlet with SOD expressed non-enzymatic antioxidants, glutathione and ascorbate which lowered hydrogen peroxide level (Diaz-Vivancos et al. 2013). In our study, it is likely that upregulation of SOD and a peroxidase is involved in scavenging ROS. Another protein, monodehydroascorbate reductase (MDHAR) which was upregulated only in B group is essential for regenerating ascorbate during ROS scavenging activity as evidenced by transgenic Brassica rapa overexpressing MDHAR and dehydroascorbate reductase (DHAR) increased glutathione and phenolics thereby alleviating redox stress leading to cold tolerance (Shin et al. 2013).

Upregulation (>10-fold) of mitogen-activated protein kinases (MAPKs) in all treatment groups might be due to MAPKs known function in facilitating host penetration during the molecular dialogue between the host and fungi (Hamel et al. 2012). The correlation between upregulation of MAPKs and sorghum biomass increase might be due to their involvement in plant hormonal responses, cell division, differentiation and regulation of antioxidant mediated defense to various stress factors (Sinha et al. 2011). Although most of ribosomal proteins are considered to be part of housekeeping proteome involved in translation with constitutive expression, some of them have been shown to be 
differentially expressed in plants due to pathogen infection and abiotic stresses. RPL3 is known to increase plant resistance to pathogenic fungi (Adam et al. 2000; Afshar et al. 2007; Kant et al. 2012; Yang et al. 2013). RPL3 was not only involved in regulation of translation but also increased antioxidant enzymes, SOD and APX in eggplant infected with Verticillium dahliae (Yang et al. 2013). It is possible that this protein has a role in mycorrhizal colonization and uptake of macroelements and $\mathrm{P}$ and $\mathrm{K}$ in root. Another protein, malic enzyme which is highly specific to malic acid provided resistance to a fungal pathogen and reduced phytotoxicity of copper in triticale plants (Garau et al. 2015; Voll et al. 2012). Solanesyl diphosphate synthase which was upregulated only in My group is required for ubiquinone synthesis in mitochondria (Ohara et al. 2010). A highly upregulated protein, PCNA has a role in DNA replication and repair (Strzalka and Ziemienowicz, 2011). Overexpression of a PCNA homolog generated UV-stress tolerant tomato plants (Gao et al. 2015), which suggests a possible role for PCNA to combat nutrient stress in sorghum.

In the current study, higher levels of some regulatory proteins correlated with uptake of specific elements and the metabolites, mucic and ferulic acids. The 26S proteasome is known to degrade proteins including damaged proteins tagged with ubiquitin and also confers tolerance to various stresses (Kurepa et al. 2009; Ueda et al. 2011). Triose phosphate isomerase levels have been reported to increase under different stresses to prevent accumulation of cytotoxic metabolites (Kaur et al. 2015). Triose phosphate isomerase is involved in glycolytic pathway, pentose phosphate pathway and carbon metabolism and distribution (Dorion et al. 2012) and sugar metabolism (Ito et al. 2003). Cysteine synthase which was upregulated in all microbial treatments is involved in fixing inorganic sulfur and sulfate assimilation in plants (Wirtz and Hell, 2006). Similar expression pattern was shown by nucleolar protein 58 which is a transcriptional regulator involved in regulating chromatin association, cell cycle, proliferation and development (González-Camacho and Medina, 2005; Moriguchi et al.et al. 2005). PAICS upregulated in all microbial treatment groups is involved in de novo purine synthesis in Arabidopsis (Senecoff et al.et al. 1996; Yamada et al. 2003) and wheat amyloplast (Dupont, 2008) but there are no reports on its association with amino acid synthesis. 
14-3-3-like protein (GF 14-C) upregulated in all three treatment groups belongs to a family of proteins involved in various biological processes and metabolic regulation, activation of transcription factors and enzymes of carbon and nitrogen metabolism (Sehnke et al. 2000; Igarashi et al. 2001). Overexpression of 14-3-3 proteins in potato altered lipid and sugar content, whereas antisense lines affected nitrate reductase activity and amino acid composition (Comparot et al. 2003; Swiędrych et al. 2002). Glutamate dehydrogenase $(\mathrm{GDH})$ which was downregulated in all three groups, showed negative correlation with sorghum biomass and has a significant regulatory function in carbon and nitrogen metabolism (Robinson et al. 1991; Ratajczak et al. 2015) that control plant growth and productivity (Dubois et al. 2003). Asparagine synthetase (AS) whose downregulation in all three treatment groups, correlated negatively with sorghum biomass, most shoot elements and some root elements, is a primary nitrogen metabolism activator and plant defense activator against pathogens (Hwang et al. 2011; Seifi et al. 2014). The common downregulated proteins among the groups showed a unique effect on amino acid biosynthesis similar to that observed with downregulated metabolites (Dhawi et al. 2015). It is likely that amino acid synthesis and nitrogen related protein upregulation with reference to all three proteins correlated negatively with element uptake as a mechanism to save energy toward biomass production in sorghum.

PIP1-1 is a member of aquaporin family of plasma membrane proteins, known to regulate water uptake and $\mathrm{CO}_{2}$ diffusion (Secchi and Zwieniecki, 2013). Aquaporins have been shown to be inhibited by heavy metals with some PIP genes downregulated under drought stress to conserve water (Niemietz and Tyerman, 2002; Jang et al. 2004). Downregulation of PIP1 genes in transgenic poplar had no significant effects on morphology, development, physiology and photosynthesis of poplar (Secchi and Zwieniecki, 2013). The 27-fold downregulation of PIP1-1 in My group and to a lesser extent in the other two treatment groups might affect TCA cycle inhibiting respiration. The PIP aquaporins have been reported to increase under water stress accompanied with upregulation of TCA cycle in grapevine (Cramer et al. 2013). Similar expression pattern was shown by hexokinase-5 which is a sugar sensor that coordinates plant sugar production, water and element uptake (Granot et al. 2013a; Granot et al. 2013b). The downregulation in our study, correlated positively with root sucrose content. However, 
hexokinase-5 reduction might be compensated by fructokinase upregulation which has a similar function.

The association of four KEGG pathways, namely, glycolysis/ gluconeogenesis, pyruvate metabolism, carbon metabolism and biosynthesis of secondary metabolites with both up and downregulated proteins would result either in the upregulation or downregulation of these pathways based on the rate limiting step in the pathway or the involvement of specific protein in alternate biological pathways. The identification of unique pathways associated with carbon fixation for common upregulated proteins and biosynthesis of amino acids and fatty acid degradation for common downregulated proteins provides clues on biochemical mechanisms operating in the systems in the current study. Protein-protein interaction analysis points to possible interactions among downregulated proteins.

\section{Conclusion}

A higher number of upregulated proteins in sorghum root were associated with PGPB, followed by $\mathrm{My}+\mathrm{B}$ and My groups, supporting significant increase in root and shoot biomass and uptake of elements in B group compared $\mathrm{My}$ and $\mathrm{My}+\mathrm{B}$ groups. Several commonly upregulated proteins showed positive correlation with biomass and uptake of elements. The upregulation of some proteins such as heat shock protein and mitogen-activated protein kinase correlated with biomass increase only, while other proteins such as those involved in ROS scavenging and DNA regulation correlated positively with element uptake only. On the other hand, monodehydroascorbate reductase (MDHAR) induced solely in B group was associated with an increase in primary elements in shoot and root, while solanesyl diphosphate synthase induced solely in My group and PAICS protein with highest upregulation in My associated groups correlated positively with uptake of elements by roots. In contrast, upregulation of proteins related to synthesis of amino acids correlated negatively with biomass or uptake of elements in sorghum. Increase in glyceraldehyde 3-phosphate and sugars as well as proteins (enzymes) associated with their metabolism in all three groups in sorghum root might indicate an increase in photosynthetic efficiency by increasing key compounds involved in carbon fixation or Calvin cycle. It is likely that the downregulation of proteins in all 
three groups was compensated with upregulation by other proteins with the same function. KEGG pathway and protein-protein interaction analysis provided information on the involvement of differentially expressed proteins in biological pathways and functional interactions. Taken together, these findings emphasize the role of PGPB in enhancing sorghum biomass and uptake of elements through metabolic pathways involving various proteins.

\section{Acknowledgements}

Scholarship by The Saudi Cultural Mission to Faten Dhawi is acknowledged. We are grateful to Dr. Erik Lilleskov (Northern Research Station, USDA Forest Service, Houghton, MI, USA) for providing comments. We thank Evan Fernandez for laboratory assistance and Mr. Steve Waling, Valentine Country Inc. for providing mycorrhiza. Funding for this work was provided by Superior Ideas to Ramakrishna Wusirika and Rupali Datta.

\section{References}

Adam, G., Mitterbauer, R., Raditschniz, A., Weindorfer, H., Glöss1, J., 2000. Introduction of a modified ribosomal protein L3 gene as a strategy to increase trichothecene toxin resistance in plants. In Proc. 2000 Natl. Fusarium Head Blight Forum, 10-12.

Afshar, A.S., Mousavi, A., Majd, A., Adam, G., 2007. Double mutation in tomato ribosomal protein L3 cDNA confers tolerance to deoxynivalenol (DON) in transgenic tobacco. Pak. J. Biol. Sci. 10, 2327-2333.

Alscher, R.G., Erturk, N., Heath, L.S.M., 2002. Role of superoxide dismutases (SODs) in controlling oxidative stress in plants. J. Exp. Bot. 53, 1331-1341.

Apel, K., Hirt, H., 2004. Reactive oxygen species: metabolism, oxidative stress, and signal transduction. Annu. Rev. Plant Biol., 55, 373-399.

Bailly, C., El-Maarouf-Bouteau, H., Corbineau, F., 2008. From intracellular signaling networks to cell death: the dual role of reactive oxygen species in seed physiology. Comptes Rendus Biologies. 331, 806-814. 
Berta, G., Copetta, A., Gamalero, E., Bona, E., Cesaro, P., Scarafoni, A., et al., 2014. Maize development and grain quality are differentially affected by mycorrhizal fungi and a growth-promoting pseudomonad in the field. Mycorrhiza 24, 161-170.

Bona, E., Scarafoni, A., Marsano, F., Boatti, L., Copetta, A., Massa, N., et al., 2016. Arbuscular mycorrhizal symbiosis affects the grain proteome of Zea mays: a field study. Scientific Rep. 6, 26439.

Comparot, S., Lingiah, G., Martin, T., 2003. Function and specificity of 14-3-3 proteins in the regulation of carbohydrate and nitrogen metabolism. J. Exp. Bot. 54, 595-604.

Cramer, G.R., Van Sluyter, S.C., Hopper, D.W., Pascovici, D., Keighley, T., Haynes, P.A., 2013. Proteomic analysis indicates massive changes in metabolism prior to the inhibition of growth and photosynthesis of grapevine (Vitis vinifera L.) in response to water deficit. BMC Plant Biol. 13, 49.

da Silva, E.C., Nogueira, R.J.M.C., da Silva, M.A., de Albuquerque, M.B., 2010. Drought Stress and Plant Nutrition, 32-41.

Dhawi, F., Datta, R., Ramakrishna, W., 2016. Mycorrhiza and heavy metal resistant bacteria enhance growth, nutrient uptake and alter metabolic profile of sorghum grown in marginal soil. Chemosphere, 157, 33-41.

Dhawi, F., Datta, R., Ramakrishna, W., 2015. Mycorrhiza and PGPB modulate maize biomass, nutrient uptake and metabolic pathways in maize grown in mining-impacted soil. Plant Physiol. Bioch. 97, 390-399.

Diaz-Vivancos, P., Faize, M., Barba-Espin, G., Faize, L., Petri, C., Hernandez, J.A., et al., 2013. Ectopic expression of cytosolic superoxide dismutase and ascorbate peroxidase leads to salt stress tolerance in transgenic plums. Plant Biotech J. 11, 976-985.

Dubois, F., Tercé-Laforgue, T., Gonzalez-Moro, M.-B., Estavillo, J.-M., Sangwan, R., Gallais, A., et al., 2003. Glutamate dehydrogenase in plants: is there a new story for an old enzyme? Plant Physiol. Biochem. 41, 565-576.

Dorion, S., Clendenning, A., Jeukens, J., Salas, J.J., Parveen, N., Haner, A.A., et al., 2012. A large decrease of cytosolic triosephosphate isomerase in transgenic potato roots affects the distribution of carbon in primary metabolism. Planta 236, 1177-1190.

Duponnois, R., Kisa, M., Assigbetse, K., Prin, Y., Thioulouse, J., Issartel, M., et al., 2006. Fluorescent pseudomonads occurring in Macrotermes subhyalinus mound structures 
decrease $\mathrm{Cd}$ toxicity and improve its accumulation in sorghum plants. Sci. Total Environ. 370, 391-400.

Dupont, F.M., 2008. Metabolic pathways of the wheat (Triticum aestivum) endosperm amyloplast revealed by proteomics. BMC Plant Biol. 8, 39.

Fukao, Y., Ferjani, A., Tomioka, R., Nagasaki, N., Kurata, R., Nishimori, Y., et al., 2011. iTRAQ analysis reveals mechanisms of growth defects due to excess zinc in Arabidopsis. Plant Physiol. 155, 1893-1907.

Gao, L., Yang, S., Zhu, Y., Zhang, J., Zhuo, M., Miao, M., et al., 2015. The tomato DDI2, a PCNA ortholog, associating with DDB1-CUL4 complex is required for UVdamaged DNA repair and plant tolerance to UV stress. Plant Sci. 235, 101-110.

Garau, G., Mele, E., Castaldi, P., Lauro, G.P., Deiana, S., 2015. Role of polygalacturonic acid and the cooperative effect of caffeic and malic acids on the toxicity of $\mathrm{Cu}$ (II) towards triticale plants ( $\times$ Triticosecale Wittm). Biol. Fert. Soils 1-10.

Gilkerson, J., Perez-Ruiz, J. M., Chory, J., Callis, J., 2012. The plastid-localized pfkBtype carbohydrate kinases FRUCTOKINASE-LIKE 1 and 2 are essential for growth and development of Arabidopsis thaliana. BMC Plant Biol. 12, 102.

Glick, B.R., 2012. Plant growth-promoting bacteria: mechanisms and applications. Scientifica doi:10.6064/2012/963401.

Gohre, V., Paszkowski, U., 2006. Contribution of the arbuscular mycorrhizal symbiosis to heavy metal phytoremediation. Planta 223, 1115-1122.

González-Camacho, F., Medina, F.J., 2005. The nucleolar structure and nucleolar proteins as indicators of cell proliferation events in plants. J. Appl. Biomed. 3,167-174.

Granot, D., David-Schwartz, R., Kelly, G., 2013a. Hexose kinases and their role in sugarsensing and plant development. Front Plant Sci. 4.

Granot, D., Kelly, G., Stein, O., David-Schwartz, R., 2013b. Substantial roles of hexokinase and fructokinase in the effects of sugars on plant physiology and development. J Exp Bot. doi: 10.1093/jxb/ert400.

Guo, J., McCulley, R.L., Mcnear, D., 2015. Tall fescue cultivar and fungal endophyte combinations influence plant growth and root exudate composition. Front Plant Sci. $6,183$. 
Hamel, L.-P., Nicole, M.-C., Duplessis, S., Ellis, B.E., 2012. Mitogen-activated protein kinase signaling in plant-interacting fungi: distinct messages from conserved messengers. Plant Cell 24, 1327-1351.

Heberle, H., Meirelles, G.V., da Silva, F.R., Telles, G.P., Minghim, R., 2015. InteractiVenn: a web-based tool for the analysis of sets through Venn diagrams. BMC Bioinformatics 16, 169.

Hwang I.S., An, S. H., Hwang, B.K., 2011. Pepper asparagine synthetase 1 (CaAS1) is required for plant nitrogen assimilation and defense responses to microbial pathogens. Plant J. 67, 749-762.

Igarashi, D., Ishida, S., Fukazawa, J., Takahashi, Y., 2001. 14-3-3 proteins regulate intracellular localization of the bZIP transcriptional activator RSG. Plant Cell 13, 2483-2497.

Ito, H., Iwabuchi, M., Ogawa, K.I., 2003. The sugar-metabolic enzymes aldolase and triose-phosphate isomerase are targets of glutathionylation in Arabidopsis thaliana: detection using biotinylated glutathione. Plant Cell Physiol. 44, 655-660.

Jaillais, Y., Chory, J., 2010. Unraveling the paradoxes of plant hormone signaling integration. Nat. Struct. Mol. Biol. 17, 642-645.

Kant, P., Gulati, A., Harris, L., Gleddie, S., Singh, J., Pauls, K., 2012. Transgenic corn plants with modified ribosomal protein L3 show decreased ear rot disease after inoculation with 'Fusarium graminearum'. Aust. J. Crop Sci. 6, 1598.

Kaur, C., Sharma, S., Singla-Pareek, S.L., Sopory, S.K., 2015. Methylglyoxal, triose phosphate isomerase, and glyoxalase pathway: implications in abiotic stress and signaling in plants. In: Elucidation of Abiotic Stress Signaling in Plants, 347-366, Springer New York.

Khan, A.G., 2005. Role of soil microbes in the rhizospheres of plants growing on trace metal contaminated soils in phytoremediation. J. Trace Elem. Med. Bio. 18, 355-364.

Kurepa, J., Wang, S., Li, Y., Smalle, J., 2009. Proteasome regulation, plant growth and stress tolerance. Plant Signal Behav. 4, 924-927.

Li, K., Ramakrishna, W., 2011. Effect of multiple metal resistant bacteria from contaminated lake sediments on metal accumulation and plant growth. J. Hazard. Mater. 189, 531-539. 
Meriga, B., Reddy, B.K., Rao, K.R., Reddy, L.A., Kishor, P.K., 2004. Aluminiuminduced production of oxygen radicals, lipid peroxidation and DNA damage in seedlings of rice (Oryza sativa). J. Plant Physiol. 161, 63-68.

Moriguchi, K., Suzuki, T., Ito, Y., Yamazaki, Y., Niwa, Y., Kurata, N., 2005. Functional isolation of novel nuclear proteins showing a variety of subnuclear localizations. Plant Cell 17, 389-403.

Ramírez, I., Estay, D., Stange, C., Cardemil, L., 2012. Superoxide dismutase is a critical enzyme to alleviate oxidative stress in Aloe vera (L.) Burm. plants subjected to water deficit. Plant Ecol. Divers. 5, 183-195.

Mittal, R., Dubey, R. S., 1991. Behaviour of peroxidases in rice: changes in enzyme activity and isoforms in relation to salt tolerance. Plant Physiol. Biochem. 29, 31-40.

Niemietz, C.M., Tyerman, S.D., 2002. New potent inhibitors of aquaporins: silver and gold compounds inhibit aquaporins of plant and human origin. FEBS Lett. 531, 443447.

Ohara, K., Sasaki, K., Yazaki, K., 2010. Two solanesyl diphosphate synthases with different subcellular localizations and their respective physiological roles in Oryza sativa. J. Exp. Bot. 61, 2683-2692.

Ratajczak, L., Ratajczak, W., Mazurowa, H., 2015. Isoenzyme pattern of glutamate dehydrogenase as a reflection of nitrogen metabolism in Lupinus albus. Acta Societatis Botanicorum Poloniae 46, 347-356.

Robinson, S.A., Slade, A.P., Fox, G.G., Phillips, R., Ratcliffe, R.G., Stewart, G.R., 1991. The role of glutamate dehydrogenase in plant nitrogen metabolism. Plant Physiol. 95, 509-516.

Secchi, F., Zwieniecki, M.A., 2013. The physiological response of Populus tremula x alba leaves to the down-regulation of PIP1 aquaporin gene expression under no water stress. Front. Plant Sci. 4, 507.

Shah, K., Kumar, R.G., Verma, S., Dubey, R.S., 2001. Effect of cadmium on lipid peroxidation, superoxide anion generation and activities of antioxidant enzymes in growing rice seedlings. Plant Sci. 161, 1135-1144. 
Sehnke, P.C., Henry, R., Cline, K., Ferl, R.J., 2000. Interaction of a plant 14-3-3 protein with the signal peptide of a thylakoid-targeted chloroplast precursor protein and the presence of 14-3-3 isoforms in the chloroplast stroma. Plant Physiol. 122, 235-242.

Seifi, H.S., De Vleesschauwer, D., Aziz, A., Höfte, M., 2014. Modulating plant primary amino acid metabolism as a necrotrophic virulence strategy: The immune-regulatory role of asparagine synthetase in Botrytis cinerea-tomato interaction. Plant Signal Behav. 9, 10.4161/psb.27995.

Senecoff, J.F., McKinney, E.C., Meagher, R.B., 1996. De novo purine synthesis in Arabidopsis thaliana (II. The PUR7 gene encoding 5 [prime]-phosphoribosyl-4-(nsuccinocarboxamide)-5-aminoimidazole synthetase is expressed in rapidly dividing tissues. Plant Physiol. 112, 905-917.

Sharma, P., Jha, A.B., Dubey, R.S., Pessarakli, M., 2012. Reactive oxygen species, oxidative damage, and antioxidative defense mechanism in plants under stressful conditions. J. Bot. doi:10.1155/2012/217037.

Shin, S.-Y., Kim, M.-H., Kim, Y.-H., Park, H.-M., Yoon, H.-S., 2013. Co-expression of monodehydroascorbate reductase and dehydroascorbate reductase from Brassica rapa effectively confers tolerance to freezing-induced oxidative stress. Mol. Cell 36, 304315.

Sinha, A.K., Jaggi, M., Raghuram, B., Tuteja, N., 2011. Mitogen-activated protein kinase signaling in plants under abiotic stress. Plant Signal Behav. 6, 196-203.

Srivastava, V., Obudulu, O., Bygdell, J., Löfstedt, T., Rydén, P., Nilsson, R., et al., 2013. OnPLS integration of transcriptomic, proteomic and metabolomic data shows multilevel oxidative stress responses in the cambium of transgenic hipI-superoxide dismutase Populus plants. BMC Genomics 14, 893.

Strzalka, W., Ziemienowicz, A., 2011. Proliferating cell nuclear antigen (PCNA): a key factor in DNA replication and cell cycle regulation. Annals Bot. 107, 1127-1140.

Swiędrych, A., Prescha, A., Matysiak-Kata, I., Biernat, J., Szopa, J., 2002. Repression of the 14-3-3 gene affects the amino acid and mineral composition of potato tubers. J. Agric. Food Chem. 50, 2137-2141. 
Szklarczyk, D., Franceschini, A., Wyder, S., Forslund, K., Heller, D., Huerta-Cepas, J., et al., 2015. STRING v10: protein-protein interaction networks, integrated over the tree of life. Nucleic Acids Res. 43, D447-452.

Ueda, M., Matsui, K., Ishiguro, S., Kato, T., Tabata, S., Kobayashi, M., et al., 2011. Arabidopsis RPT2a encoding the $26 \mathrm{~S}$ proteasome subunit is required for various aspects of root meristem maintenance, and regulates gametogenesis redundantly with its homolog, RPT2b. Plant Cell Physiol. 52, 1628-1640.

Voll, L.M., Zell, M.B., Engelsdorf, T., Saur, A., Wheeler, M.G., Drincovich, M.F., et al., 2012. Loss of cytosolic NADP-malic enzyme 2 in Arabidopsis thaliana is associated with enhanced susceptibility to Colletotrichum higginsianum. New Phytol. 195, 189202.

Wirtz, M., Hell, R., 2006. Functional analysis of the cysteine synthase protein complex from plants: structural, biochemical and regulatory properties. J. Plant Physiol. 163, 273-286.

Yamada, K., Lim, J., Dale, J.M., Chen, H., Shinn, P., Palm, C.J., et al., 2003. Empirical analysis of transcriptional activity in the Arabidopsis genome. Science 302, 842-846.

Yang, L., Xie, C., Li, W., Zhang, R., Jue, D., Yang, Q., 2013. Expression of a wild eggplant ribosomal protein $\mathrm{L} 13 \mathrm{a}$ in potato enhances resistance to Verticillium dahliae. Plant Cell Tiss. Organ Cult. 115, 329-340.

Table 1. List of KEGG Pathways affected by common upregulated and downregulated proteins in sorghum root.

\begin{tabular}{|l|l|c|l|}
\hline Upregulated proteins & $\begin{array}{l}\text { count in } \\
\text { gene set }\end{array}$ & $\begin{array}{l}\text { false discovery } \\
\text { rate }\end{array}$ \\
\hline pathway ID & pathway description & 3 & 0.00035 \\
\hline 10 & Glycolysis / Gluconeogenesis & 2 & 0.00622 \\
\hline 620 & Pyruvate metabolism & 2 & 0.00563 \\
\hline 710 & Carbon fixation in photosynthetic organisms & 4 & 0.00711 \\
\hline 1110 & Biosynthesis of secondary metabolites & 4 & $6.37 \mathrm{E}-05$ \\
\hline 1200 & Carbon metabolism & \multicolumn{2}{l}{} \\
\hline \multicolumn{5}{|l|}{ Downregulated proteins } & 3 & 0.00418 \\
\hline 10 & Glycolysis / Gluconeogenesis \\
\hline
\end{tabular}




\begin{tabular}{|l|l|c|l|}
\hline 71 & Fatty acid degradation & 2 & 0.00742 \\
\hline 620 & Pyruvate metabolism & 3 & 0.000982 \\
\hline 1110 & Biosynthesis of secondary metabolites & 10 & $2.12 \mathrm{E}-06$ \\
\hline 1200 & Carbon metabolism & 6 & $1.94 \mathrm{E}-06$ \\
\hline 1230 & Biosynthesis of amino acids & 6 & $2.00 \mathrm{E}-06$ \\
\hline
\end{tabular}

String web site (string-db.org/cgi/network.pl) was used to generate this data. Metabolic pathways affected by downregulated proteins but not by upregulated proteins are shown in italics. 


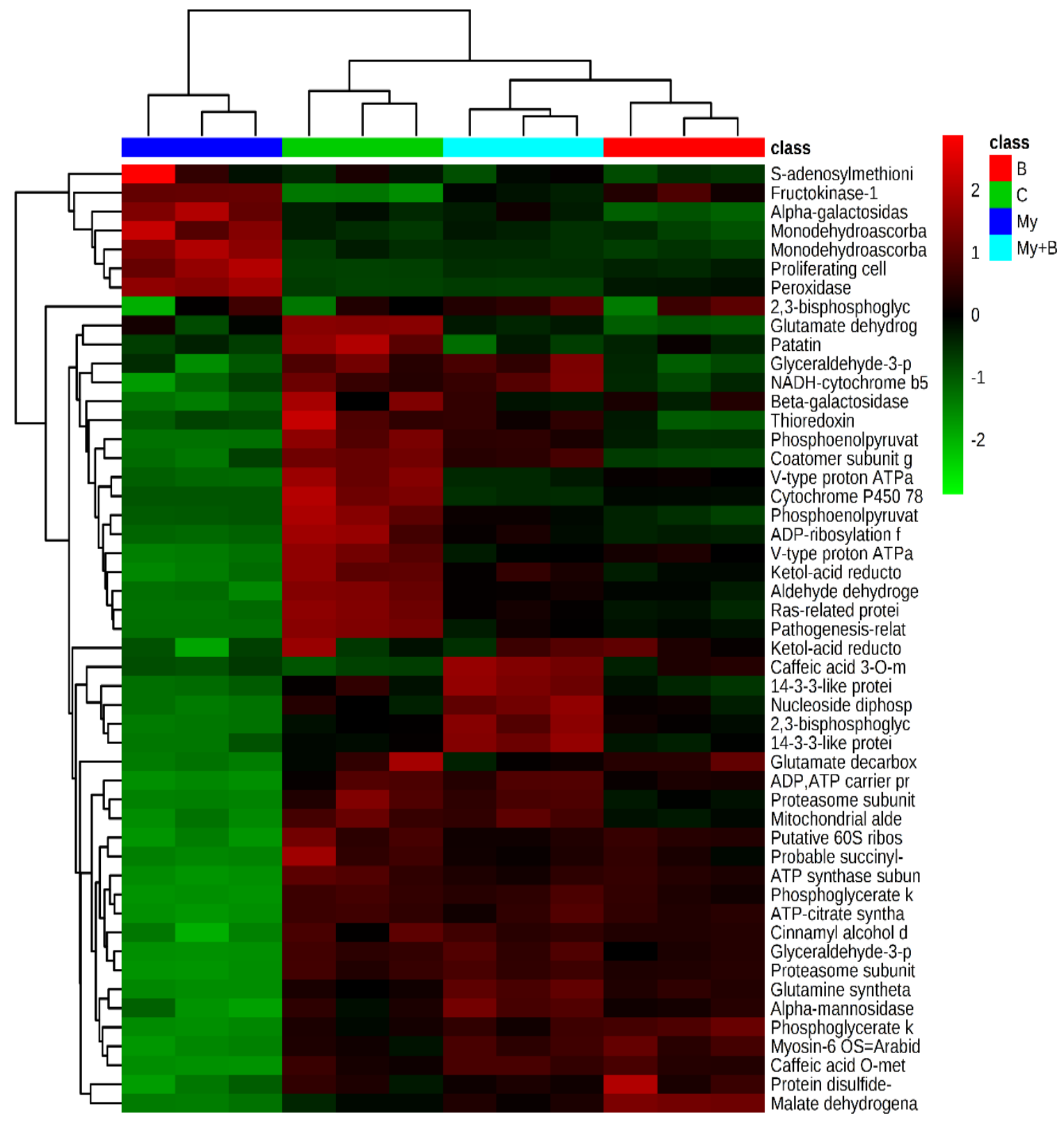

Fig. 1. Heat map showing top 50 significant plant proteins identified in sorghum roots subjected to different treatments (B, My, My+B) and control (C). Heat map was generated using hierarchical clustering with hclust function in the STAT package. The similarity was measured using Euclidean distance and Ward's linkage clustering algorithms. The names of differentially expressed proteins are shown on the X-axis on the right. The red color in the heat map indicates upregulation of proteins and green indicates their downregulation. 


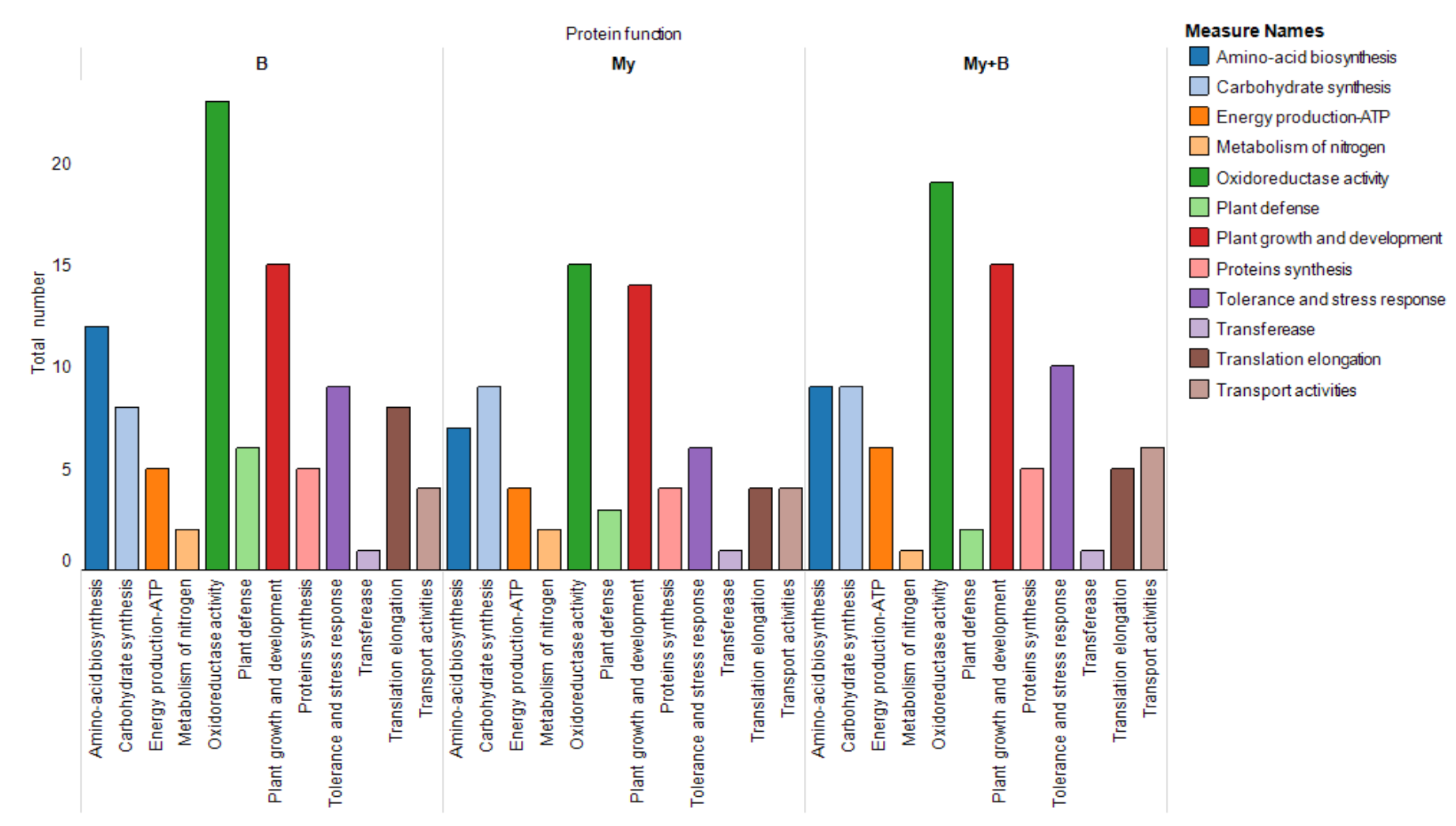

Fig. 2. Functional classification of proteins in B, My and My+B groups. The figure was generated using Tableau software (http://www.tableau.com). The functions of the proteins were assigned using Uniprot (www.uniprot.org). Total number of proteins belonging to each functional group are shown on $\mathrm{X}$-axis. 


\section{Fold change}

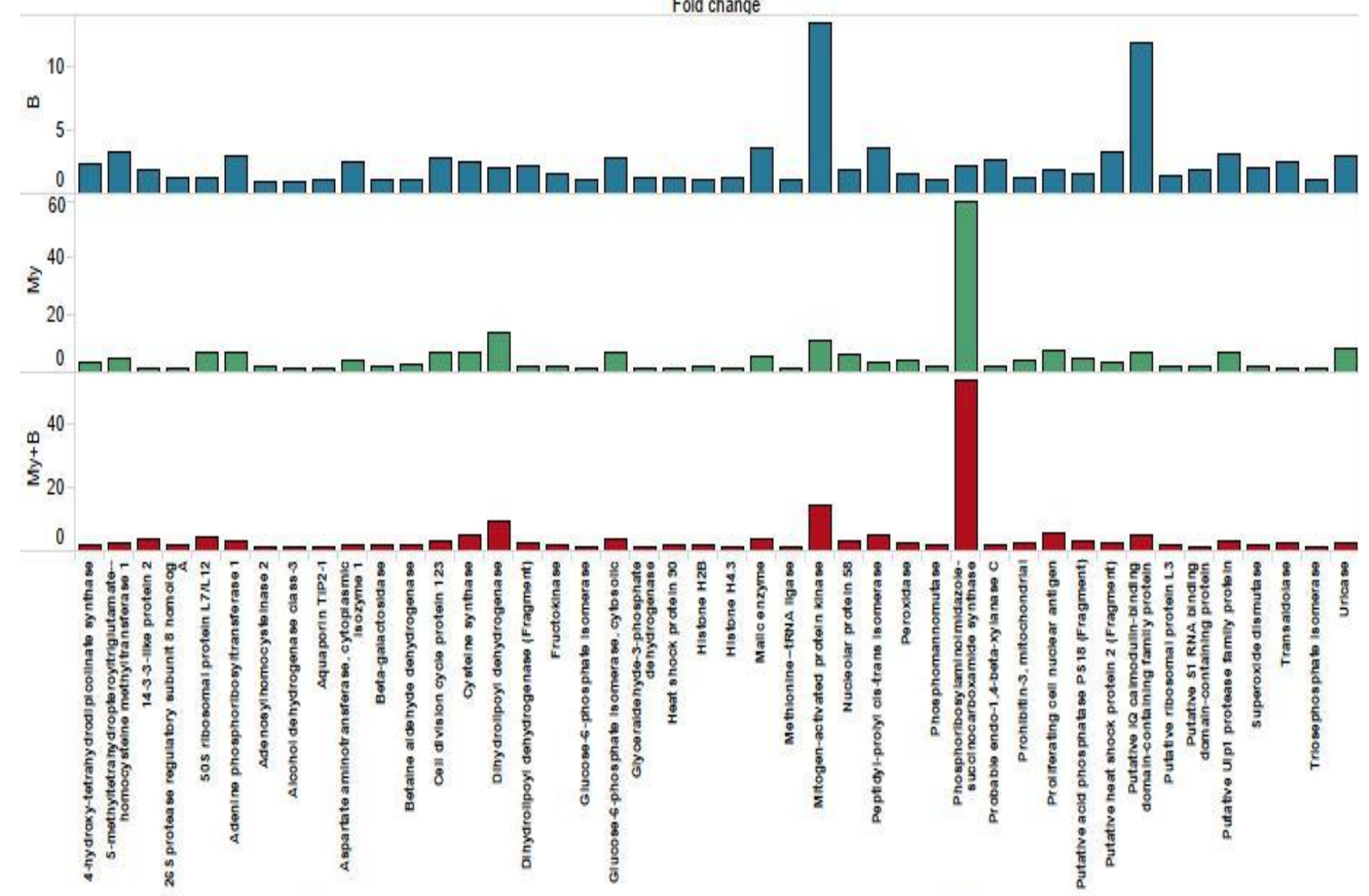

Fig. 3. Quantitative analysis showing fold change of 44 proteins upregulated in all three groups $(B, M y$ and $M y+B)$ in comparison to the control group. Fold changes of specific proteins are shown on $\mathrm{X}$-axis and names of proteins are shown on $\mathrm{Y}$ axis. The figure was generated as described in figure 2 . 


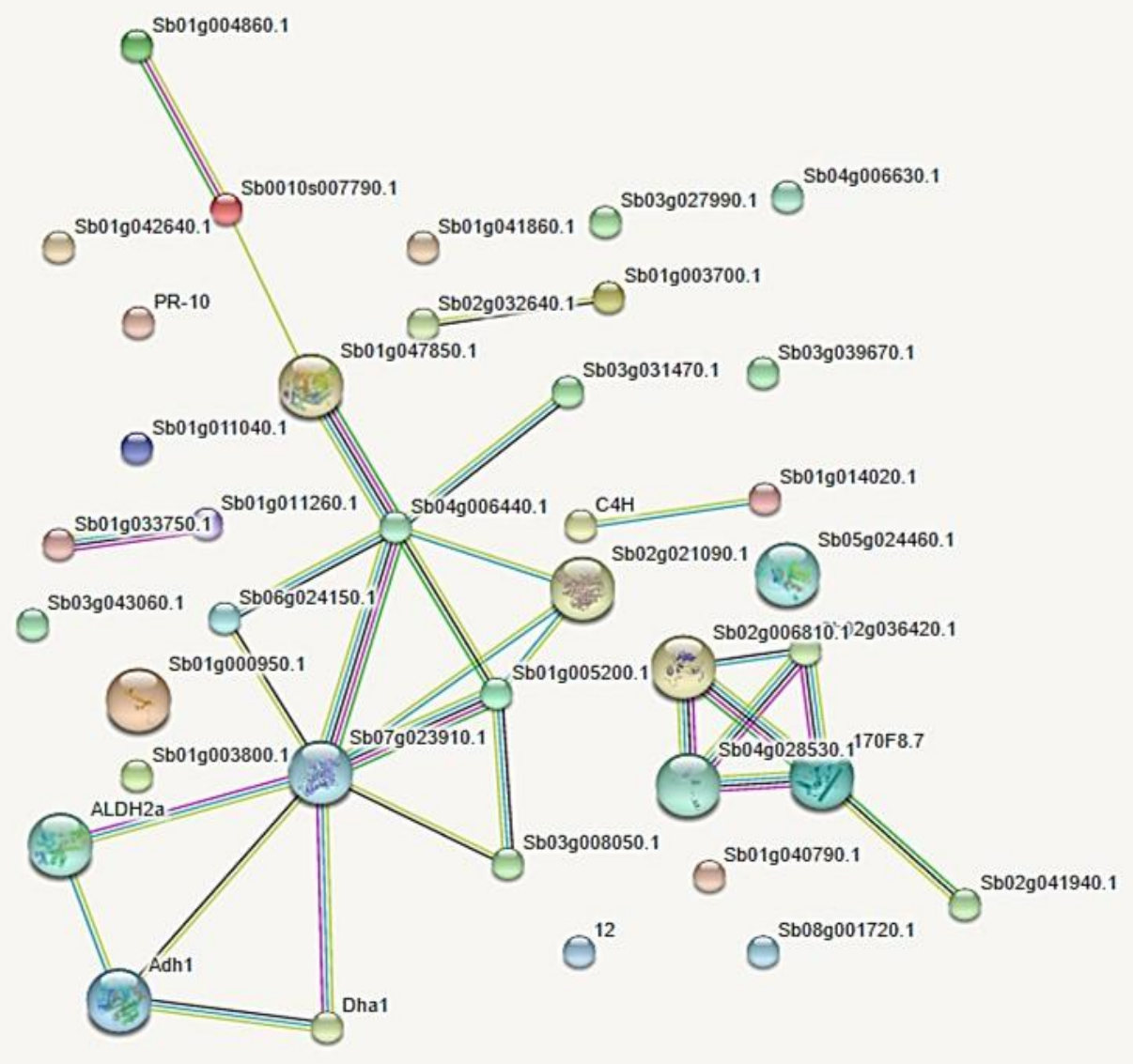

Fig. 4. Common downregulated sorghum root proteins associated with biological pathways subjected to protein-protein interactions analysis using STRING. Downregulated proteins showing interactions with other proteins in the major network are Sb01g004860: Thioredoxin, Sb0010s007790: Glutathione peroxidase, Sb01g047850: Aconitate hydratase, Sb04g006440: Citrate synthase, Sb06g024150: Glutamate dehydrogenase, Sb07g023910: Malate dehydrogenase 2, Sb03g008050: Fructose bisphosphate aldolase, Sb01g005200: Pyruvate kinase, Sb02g021090.1: Phosphoenolpyruvate carboxylase, Sb03g031470: ATP synthase subunit $\beta$, ALDH2a: Mitochondrial aldehyde dehydrogenase, Adh1: Putative alcohol dehydrogenase, and Dha1 (Sb02g025790): Aldehyde dehydrogenase. The second network has Sb02g006810: 40S ribosomal protein S12, Sb04g028530: 40S ribosomal protein S8, S8170F8.7: Putative 60S ribosomal protein, Sb02g036420: Elongation factor 1 $\alpha$, and Sb02g041940: Elongation factor Ts. Three interactions involving only two proteins each are $\mathrm{C} 4 \mathrm{H}$ (Sb02g010910): Cinnamic acid 4-hydroxylase and Sb01g014020: Phenylalanine ammonia lyase; Sb01g033750: Proteasome subunit $\alpha$ and Sb01g011260: Proteasome subunit $\beta$; Sb02g032640: NADPH Cytochrome P450 reductase and Sb01g003700: Methylene tetrahydrofolate reductase. 